Military Technical College Kobry El-Kobbah, Cairo, Egypt

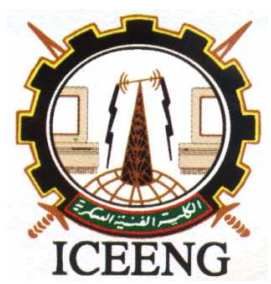

\author{
$6^{\text {th }}$ International Conference \\ on Electrical Engineering \\ ICEENG 2008
}

\title{
Diagnostic comparison of water tree growth in XLPE insulated power cables produced in GCC countries
}

By
A.A. Al-Arainy*
M.I. Qureshi*
N.H. Malik*
M.N. Saati**
O.A. Al-Nather***
S. Anam****

\section{Abstract:}

Electric power utilities and industrial units in Saudi Arabia are extensively using XLPE cables produced in the Arabian Gulf region. Due to prevailing high temperatures and high sulphate content in the soil coupled with high sub surface water table, these cables have suffered serious breakdowns in a short time span after their installation. This investigation was undertaken to compare the resistance to water treeing in of $\mathrm{kV}$, XLPE insulated cables of four regional manufacturers that are producing these cables according to international standards. In first stage, aging was carried out under high electric stress and temperature cycling in the presence of deionized water. After 120 days of this aging, their remaining dielectric strength was determined. These results showed poor performance of one type while the others exhibited close values. In the second stage, the cables were aged in the presence of aqueous ionic solution of $\mathrm{CuSO}_{4}$ for aging periods that lasted in the range of 500 to 1500 hours. Water tree population and tree lengths were compared and their number and length distributions were subjected to statistical Weibull and Log-normal models. Water tree population and their length distributions were found to fit better on Log-normal model. This suggests that the most likely mechanism for environmentally induced water tree degradation is electromechanical fatigue based. On the other hand, water tree density as a function of aging period was found to obey a log-linear relation. These data also show that water tree density in these cables as evaluated in the second stage of aging, comparatively varies significantly and leads to better segregation and selection of the cable that will serve the best in such environmental conditions. 


\section{Index Terms:}

XLPE power cables, Accelerated multifactor aging, aqueous ionic $\mathrm{CuSO}_{4}$ solution, retained dielectric strength, water tree statistics, water treeing

\footnotetext{
* College of Engineering, King Saud University, Riyadh, Saudi Arabia

** Riyadh Group of Cables, Riyadh, Saudi Arabia

*** King Abdualaziz City for Science and Technology, Riyadh, Saudi Arabia

**** $\quad{ }^{1}$ Saudi Electricity Company, Riyadh, Saudi Arabia
}

\section{Introduction:}

Electric power utilities and industrial units are extensively using XLPE cables that are being produced in the Gulf Cooperation Council (GCC) countries. Medium voltage oil filled paper insulated cables are rapidly being replaced with their XLPE-insulated counter parts due to their excellent properties and maintenance-free nature. In the last two decades, a number of local cable manufacturers have emerged in the GCC-market and are playing a vital role in the development of underground electric power networks in this region. However, despite several merits of XLPE insulated cables, the utilities in Saudi Arabia are experiencing premature failures. This is attributed mainly to high prevailing temperatures and high sulphate content in the sub-soil level coupled with high water table in coastal regions [1,2]. These outages are, of course, a matter of gross concern for power utilities and the question as how to address this problem and predict the performance of these cables has assumed great importance $[3,4]$. This investigation was undertaken initially to compare the performance of these cables when subjected to long term accelerated aging as per the criteria set in AEIC-CS5-1994 [5]. $15 \mathrm{kV}$, XLPE insulated cables of four major regional manufacturers that are producing these cables according to international standard were compared. The aging was carried out under high electric stress and temperature cycling in the presence of deionized water. After 120 days of this aging, the retained dielectric strength of these cables was determined. This aging test showed poor performance of one cable while three others exhibited closer values. Even the water tree count did not lead to clear comparison. Therefore, a second strategy was adopted. In this case, the accelerated aging was carried out in the presence of aqueous ionic solution of $\mathrm{CuSO}_{4}$ for aging periods that lasted in the range of 500 to 1500 hours. Water tree population and tree lengths were investigated and compared in this stage. The tree number and length distribution were subjected to 
statistical Weibull and Log-normal models. Water tree density as a function of aging period was also determined and analyzed. Detailed results address the implications of both types of aging techniques applied and are discussed in detail.

\section{Experimental Techniques}

\subsection{Cable-Samples Tested}

XLPE insulated power cables that are abundantly being used by local electrical power utilities and produced by four major XLPE cable manufacturers based in GCC countries were investigated in this study. All four types of cables were produced to the same specifications and the samples were acquired from the local market. All cables were rated $15 \mathrm{kV}$, and were 3 core, $185 \mathrm{~mm}^{2}$ copper conductor crosss-section, XLPE insulation, copper screen, steel wire armour, and had an outer sheath of PVC. These cable samples are assigned here-to-forth the symbols as A,B,C and D.

\subsection{Aging Techniques Investigated}

In this investigation the accelerated aging was carried out in two different ways to compare the parameters like retained dielectric strength, types of water trees produced and their statistics. In the first technique, the cables were aged as per AEIC protocol [5] following the "preset aging time method". Each cable type was desheathed and six single core samples, each of $\sim 9 \mathrm{~m}$ length were prepared for test purpose. Prior to accelerated water tree test (AWTT) aging program, these cables were subjected to heat cycle aging at $130{ }^{\circ} \mathrm{C}$ for two weeks. In AWTT, cable conductors were injected with deionized water while the cable's outer earth shield was surrounded by water filled in PVC pipes/tanks set up. This is a modified version of AWTT protocol of AEIC-CS5 [5] and is illustrated elsewhere [6]. Cable insulation was stressed by a voltage of $3 \mathrm{U}_{\mathrm{o}}$ where $\mathrm{U}_{\mathrm{o}}$ is the rated line to neutral voltage of the cable. The conductor was heated and load cycled by passing current through it. All of the 24 samples from four manufacturers were thus subjected to multi-factor aging in one single batch. After completion of 120 days of aging, these cables were removed and subjected to breakdown tests to determine their retained dielectric strength as compared to those of new unaged samples. This testing was carried out as per high voltage time test (HVTT) protocol illustrated in clause M.2.1 of AEIC [5].

Six samples from each type (new as well as aged) were subjected to HVTT to get their breakdown values. These values were then plotted on Weibull-probability paper to get characteristic value $(62 \%)$ of the breakdown $\left(\mathrm{V}_{\mathrm{c}}\right)$. In addition, 25 insulation wafers of $0.4 \mathrm{~mm}$ thickness from each aged cable were also cut, stained and subjected to microscopic examination in order to evaluate and compare water tree statistics in these 
samples. The studied tree parameters included, type of water tree (bow-tie or ventedtype), the maximum length $\left(\mathrm{L}_{\mathrm{m}}\right)$ and the number density $\left(\mathrm{n}_{\mathrm{t}}\right)$ i.e. number of bow-tie trees per cubic millimeter of insulation volume.

In the second technique of aging, the cable's samples (A,B,C, and D) were immersed in aqueous ionic solution of 0.01 molar concentration of $\mathrm{CuSO}_{4}$ to evaluate the impact of this salt which is abundant in the sub-surface soil of most of the regions in Arabian peninsula. For each cable type, four meter long single core samples were cut and their copper screens were removed. Around 1.00 meter length of insulation semiconducting screen was stripped away from each cable at both ends and $35 \mathrm{kV}$ rated stress cones were inserted. Each sample was placed inside a grounded and covered stainless steel tank which was $1.0 \mathrm{~m}$ long, $20 \mathrm{~cm}$ wide and of $25 \mathrm{~cm}$ in height and each filled with the $\mathrm{CuSO}_{4}$ ionic solution. Fig. (1) illustrates the complete aging set up. The cable samples were connected in parallel and energized under $60 \mathrm{~Hz}$ at $3 \mathrm{U}_{\mathrm{o}}$. The fluid was circulated in closed loop with the help of a microprocessor controlled thermal fluid circulator. During this aging, temperature of $50^{\circ} \mathrm{C}$ was maintained for 6 hours, while it was kept switched off for the subsequent 18 hours period each day. The cable conductor was not subjected to any current cycling in this case. Using these stress parameters, the cables were aged in batches for 500, 800, 1000 and 1500 hours. After the end of each predefined aging period, 25 number $0.4 \mathrm{~mm}$ thick insulation wafers from each cable sample were microtomed and dyed for microscopic investigations of water trees.

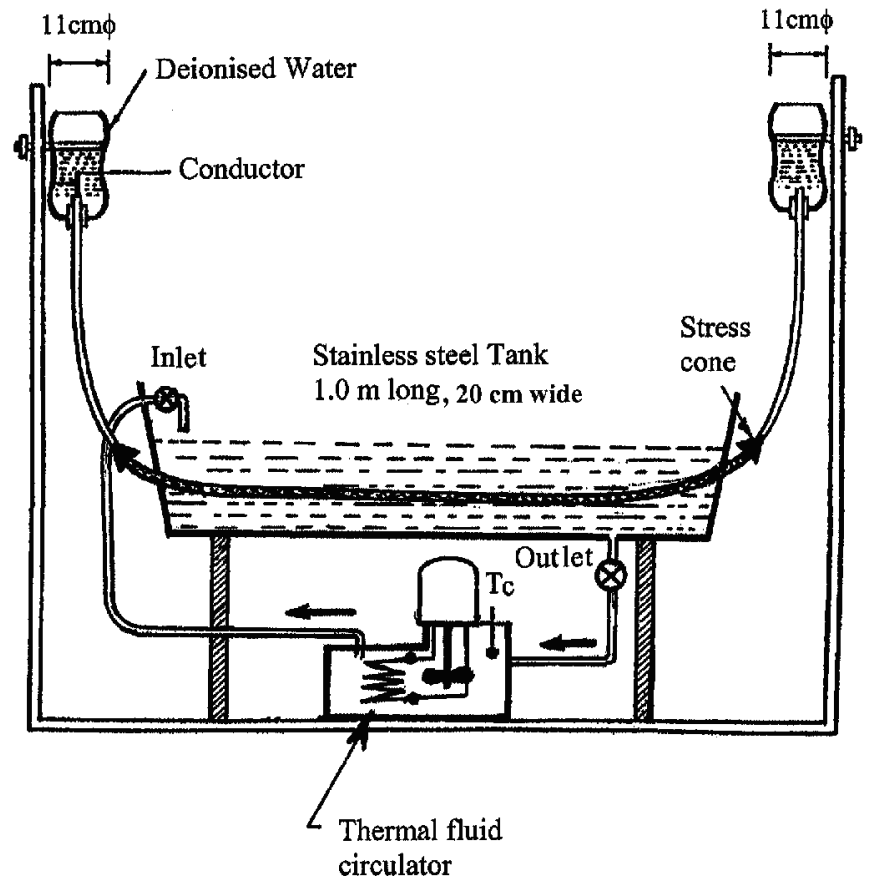

Fig. (1): Cable aging set up used to evaluate the impact of degradation of a cable sample. 


\section{Results And Discussion}

\subsection{Effect of Aging as per AEIC Protocol}

Fig. (2 a\&b) display the comparison of variation of breakdown voltage for the new and aged cable samples (type A) that was obtained following the procedure of HVTT as per AEIC-protocol [5]. These results are drawn on Weibull probability plot with 90\% confidence limit bounds which clearly show that breakdown probability of polymeric cables follows Weibull statistical model for new as well as aged cables which is consistent as reported in literature [4,7]. Similar are the results for the other cable samples, as well. However, the value of $\mathrm{V}_{\mathrm{c}}$ for old cables are much lower than the new cable samples. Table (1) compares the $62 \%$ of characteristic breakdown voltage $\left(\mathrm{V}_{\mathrm{c}}\right)$ for four new as well as aged cables. These results clearly show that cable type A has the highest breakdown voltage among the new samples. However, it also shows the highest reduction in the breakdown voltage due to AWTT aging which leads to severe water treeing in these cables. Cable type D has the lowest breakdown voltage among the four, but it also exhibits lowest reduction in retained dielectric strength due to accelerated aging. In general, cable $\mathrm{A}$ is left with retained breakdown strength of $~ 59 \%$ while the other three samples exhibit its value well above $80 \%$. In order to further segregate this outcome, the propensity toward water treeing was also examined. Only few vented trees were observed in all samples, which shows the cleanness of the inner and outer semi-conducting screen materials used in the production of these cables. However, large population of bow-tie trees emerged in the main insulation's matrix of these cables. Fig. (3) displays the presence of several small and large bow-tie trees in an aged cable sample. One of these trees was found to bridge almost $32 \%$ the total insulation thickness of cable. Since it is the longest water tree which represents weakest link in the insulation that can lead to catastrophic breakdown, their number density was evaluated, but it also did not lead to segregation of a better cable among the four compared. It is clear that this type of aging does not lead to a clear evidence based on which one can select the cable sample that will perform better in the real practical life. 


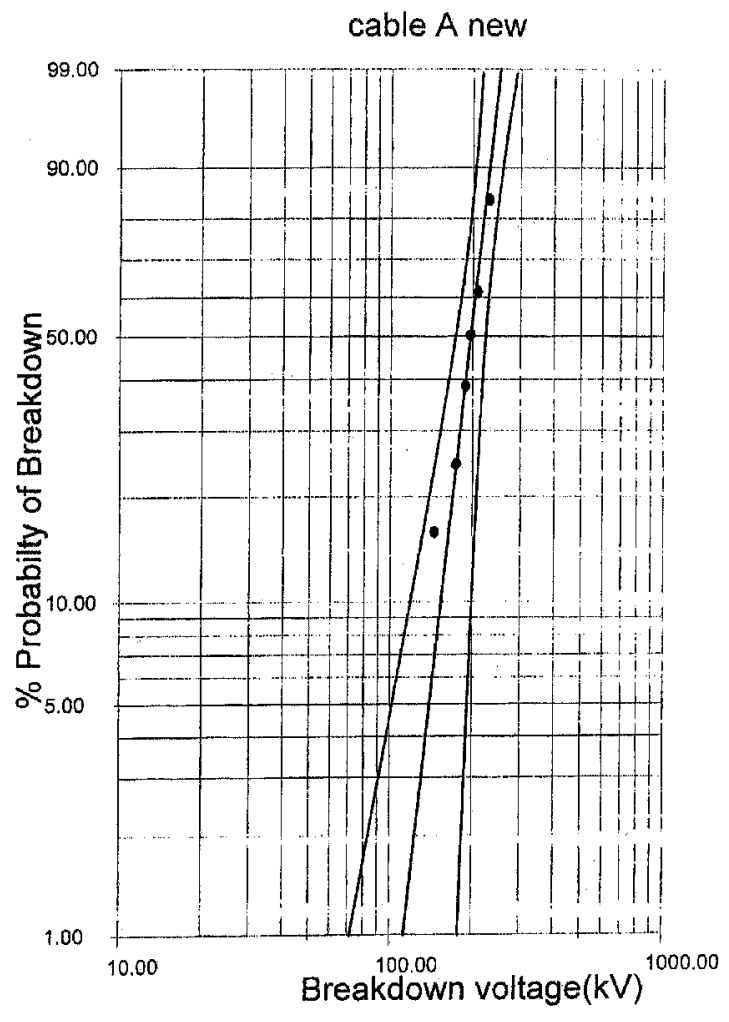

(a)

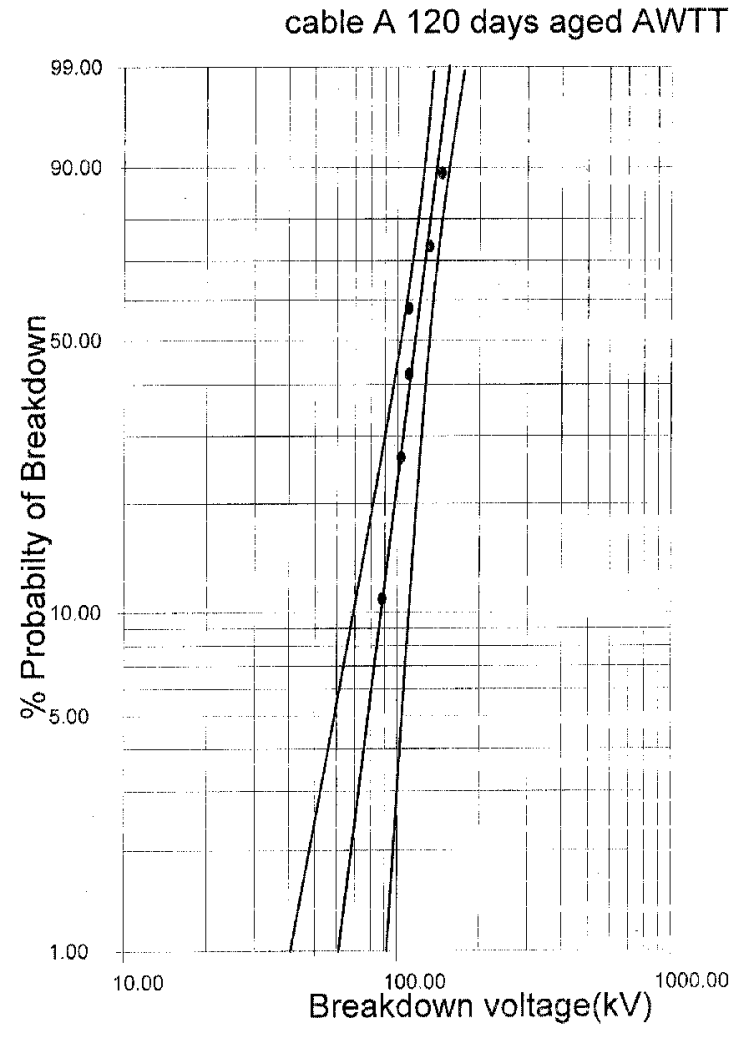

(b)

Fig. (2): Weibull probability plot for breakdown voltages of: (a) new; and (b) old cable samples of type (A).

Table (1): Comparison of 62\% characteristics breakdown voltage $V_{c}$ for four types of cables.

\begin{tabular}{|c|c|c|c|}
\hline \multirow[b]{2}{*}{ Cable Type } & \multicolumn{2}{|c|}{$\mathrm{V}_{\mathrm{c}}(\mathrm{kV})$} & \multirow{2}{*}{$\begin{array}{l}\text { Ratio of } V_{c} \text { of } \\
\text { aged to } V_{c} \text { of } \\
\text { new cable }(\%)\end{array}$} \\
\hline & $\begin{array}{l}\text { New } \\
\text { Cable }\end{array}$ & $\begin{array}{c}120 \text { days AWTT } \\
\text { aged cables. }\end{array}$ & \\
\hline A & 206 & 122.6 & 59 \\
\hline B & 160 & 135.0 & 85 \\
\hline $\mathrm{C}$ & 170.0 & 137 & 82 \\
\hline $\mathrm{D}$ & 157.5 & 144.1 & 91 \\
\hline
\end{tabular}




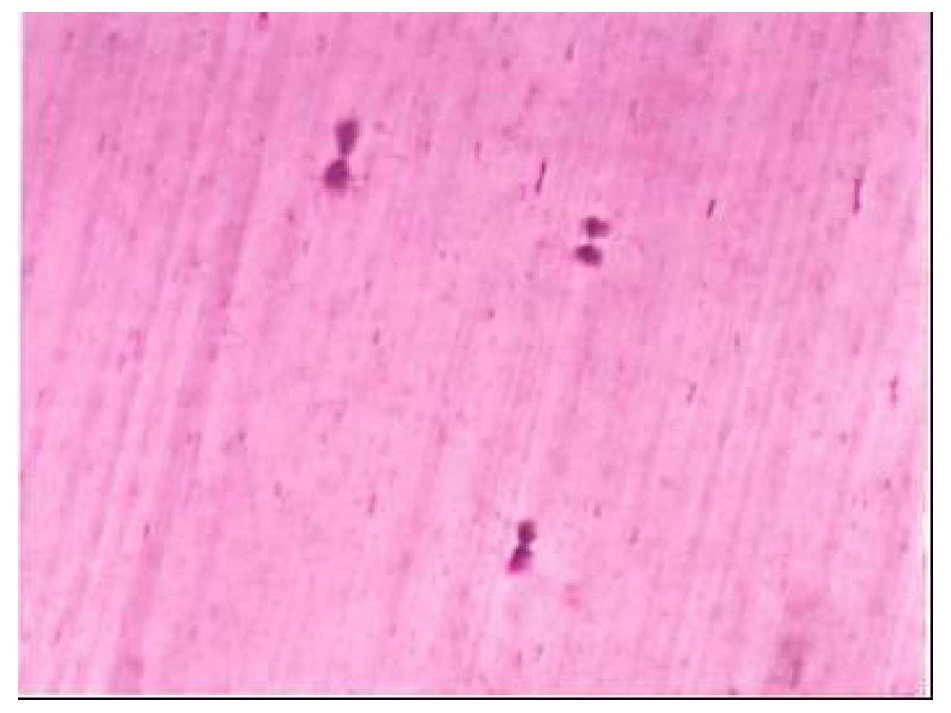

Fig. (3): Several long and short bow-tie water trees in cable 'C'.

\subsection{Impact of Aging in Aqueous Ionic Solution}

In this case, after completion of each aging period, the total number of water trees as well as tree length distributions of these trees and water tree's number density were measured. The length of every tree detected $(\geq 10 \mu \mathrm{m})$ was measured. Table (2) summarizes the value of ' $n_{t}$ ' as a function of aging time. It is gratifying to note here that this aging has imparted very distinct effect of water treeing in each cable. The cable that will perform the best in such environment can easily be segregated based on these results. Fig. (4) displays a plot of this data and it is clear that it follows the equation:

$$
\mathrm{n}_{\mathrm{t}}=\mathrm{A} \log _{10}(\mathrm{t})-\mathrm{B}
$$

where, $\mathrm{n}_{\mathrm{t}}=$ water tree density (number of bow-tie trees, and $\mathrm{t}=$ aging time (hours).

Table (2): Effect of aging time on water tree density for cable type B.

\begin{tabular}{|c|c|}
\hline $\begin{array}{c}\text { Aging Time } \\
\text { (hours) }\end{array}$ & $\begin{array}{c}\text { Tree Density } \\
\text { (number } / \mathrm{mm}^{3} \text { ) }\end{array}$ \\
\hline 500 & 0.0581 \\
\hline 800 & 2.3 \\
\hline 1000 & 3.48 \\
\hline 1500 & 5.2 \\
\hline
\end{tabular}




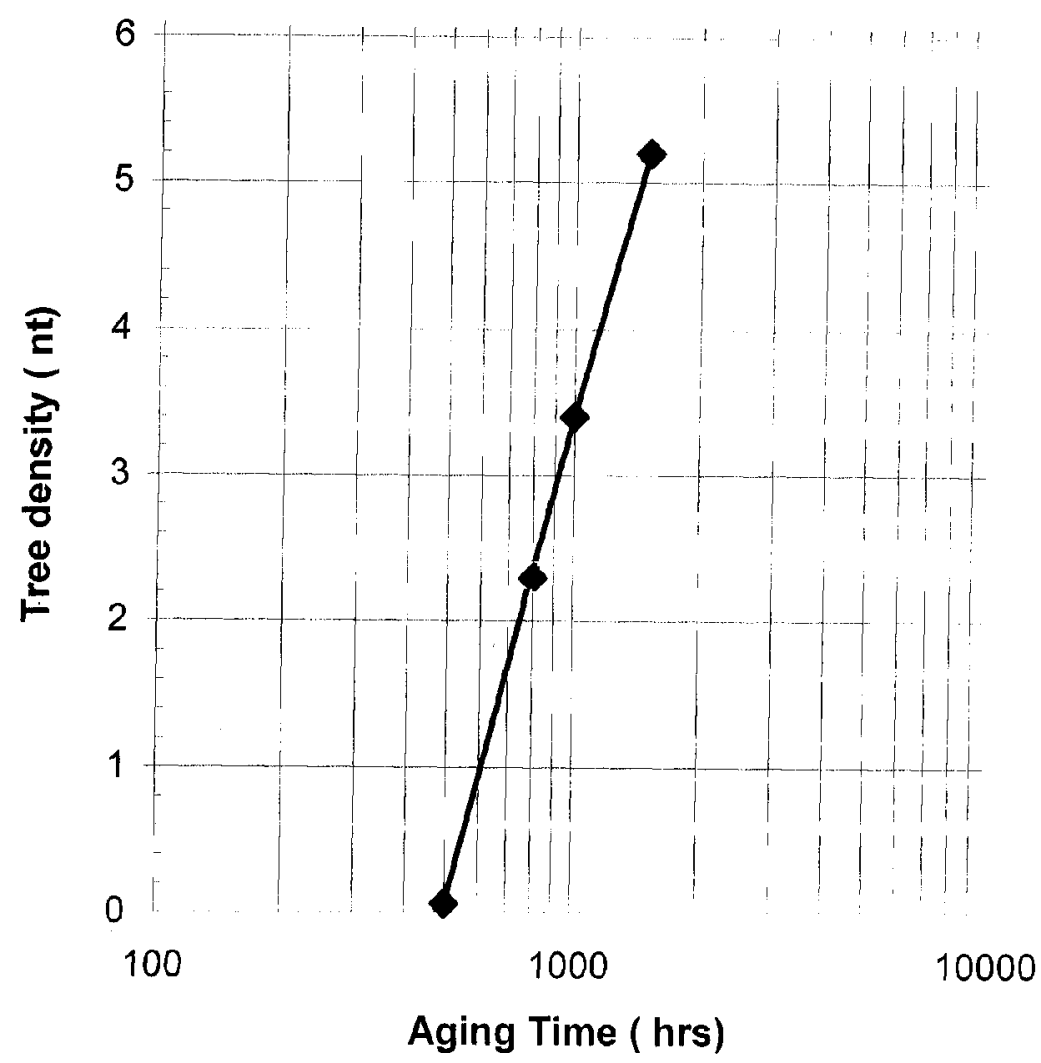

Fig. (4): Variation of $n_{t}$ versus aging time for one of the tested cables.

The tree count data scanned and collected from 25 slices of each type of cable was further investigated for statistical variations. Almost all results plotted on histograms showed skewed distributions. There exist several theoretical distributions that may be applied to analyze such data. In all cases, the best choice depends on the statistical criteria, while the goodness of fit on a particular distribution model represents certain physical phenomena. It is therefore imperative to understand, not just the resulting statistics but also the physics and the embedded technical reasons, as well. Inception and growth of water trees is a random statistical process and must be treated statistically and understood physically. There is now general consensus among the researchers that water treeing phenomena can range from predominantly electromechanical to essentially electro-chemical in nature. Controversy still exists and the actual controlling process that initiates these weak spots in insulation needs yet to be delineated. On this regard several researchers have applied the asymptotic extreme value statistical analysis, since the initiation of water tree depends on one of the processes described above. However, controversy exists on the best fit either on log-normal or Weibull model of tree length distribution [7-10]. In this context, the results obtained including the characteristic parameters were evaluated for the two types of statistical distributions. Here the main purpose of subjecting the tree lengths to these statistical models is to 
determine whether the tree growth is dominated by rapid propagation from weak sites (Weibull model) or whether it is the result of a fatigue based mechanism in which several factors act cooperatively (LN-model).

For all aging times, when the tree length data were subjected to a Weibull statistical distribution, a poor fit was obtained which indicates that water tree lengths do not obey Weibull distribution. Fig. (5a) displays such a selected case. However, an excellent fit was obtained for log-normal distribution plots for all cases studied. Fig. (5b) exhibits data of Fig. (5a) plotted on L-N plot. LN-plots of all other aged cables (not shown here) display similar trend. Thus, it can be concluded that water tree initiation and growth is dominated by a mechanism in which many factors cooperate, such as in fatigue failure of components.

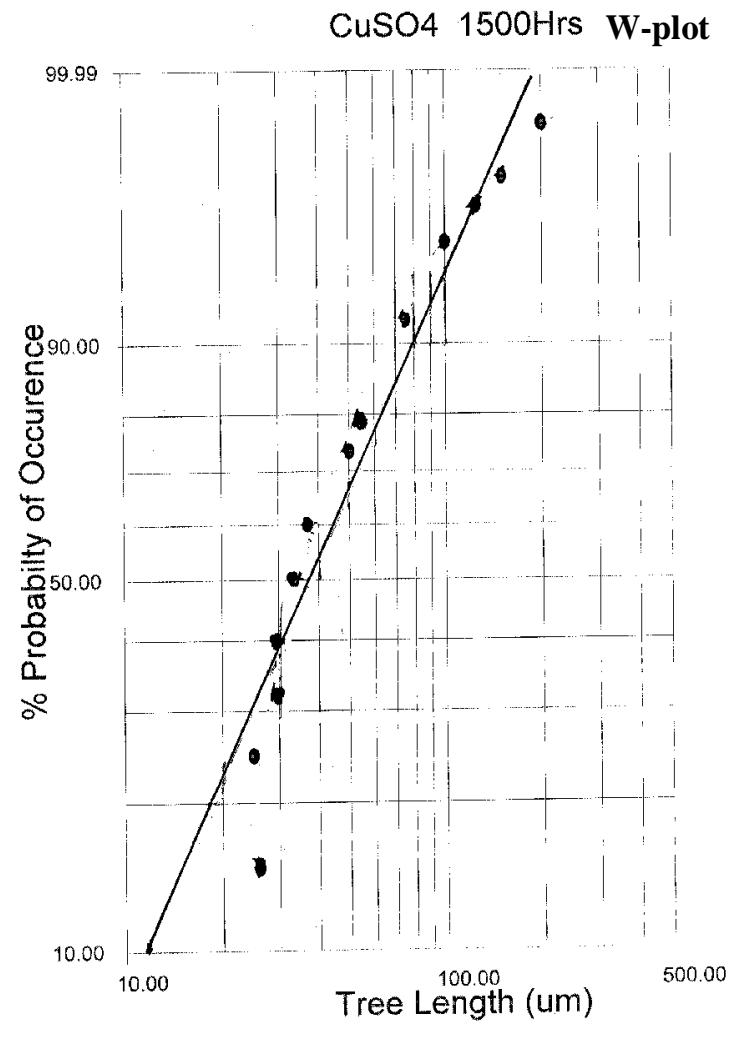

(a)

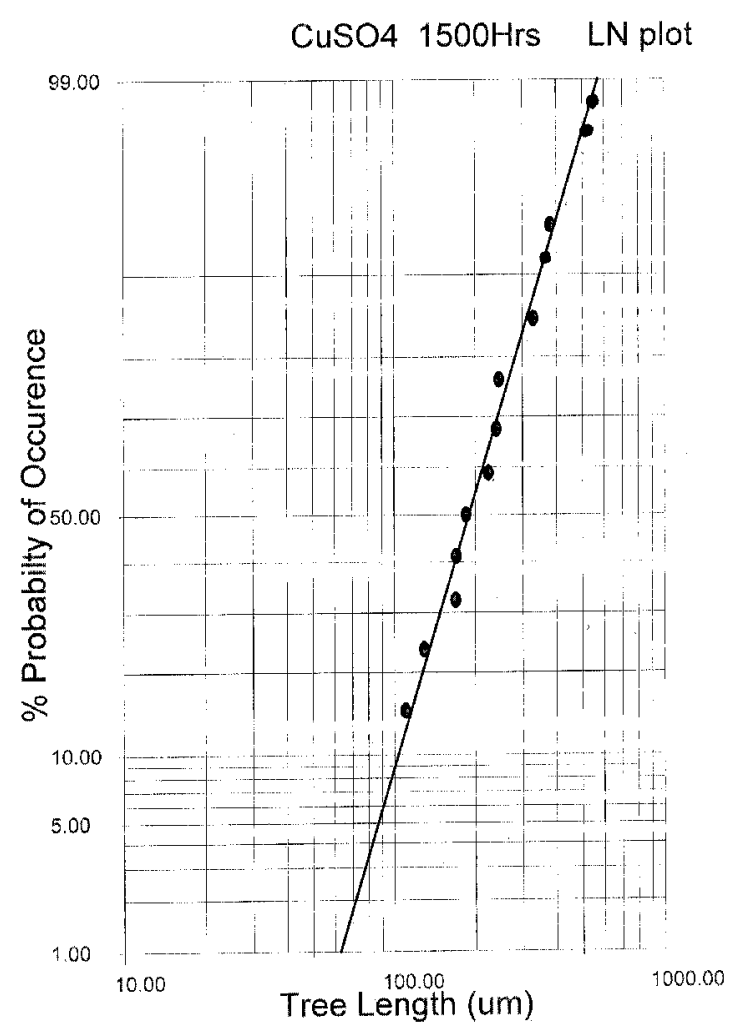

(b)

Fig. (5): (a) Weibull distribution plot of bow-tie tree length data for aged cable $B$ for 1500 hours aged under 0.01 mole $\mathrm{CuSO}_{4}$ solution maintained under $3 \mathrm{U}_{o}$ voltage, (b) Log-normal distribution plot of data given in Fig. (5-a). 
These studies substantiate this attribute which was forwarded earlier by Al-Arainy et al. [8] while reporting water treeing in cables aged under aqueous ionic solution of sodium chlorides. These results therefore confirm this mechanism related to water treeing, which is not yet fully understood despite the efforts being exerted in several research centers worldwide. These results also show that water tree density as evaluated in the later technique of aging leads to better segregation and selection of the cable that will serve the best in such harsh local sub-surface soil conditions.

\section{Conclusions}

This experimental investigation leads to the following:

1. Based on 120 days of AWTT aging as per AEIC protocol, the retained dielectric strength as well as tree counts shows poor comparative performance of investigated cables.

2. An alternate quicker and economical technique is the accelerated aging in presence of aqueous ionic solution of $\mathrm{CuSO}_{4}$ for aging periods of $\sim 1000$ hours and comparison of water tree density so produced. This method also revealed that water tree density varies with aging time that follows a log-linear relation.

3. The statistical behavior of water tree lengths follows log-normal distribution, which shows that their initiation is controlled by fatigue based mechanisms.

\section{Acknowledgement}

This work was supported by King Abdulaziz City for Science and Technology (KACST), Riyadh, Saudi Arabia, through their Research Grant \# A-3-2.

\section{References}

[1] I.F. Hodgson et al., Proc. of $2^{\text {nd }}$ Symposium on Geotechnical Problems in Saudi Arabia, Riyadh, pp. 164-175, 1989.

[2] A.A. Al-Arainy, N.H. Malik, M.I. Qureshi, and M.N. Saati, "The Performance of Strippable and Bonded Screened Medium-Voltage XLPE Insulated Cables under Long-Term Accelerated Aging", IEEE Trans. on Power Delivery, Vol. 22, No. 2, pp. 744-751, 2007.

[3] A.A. Al-Arainy, N.H. Malik, M.I. Qureshi and N. Al-Saati, "Investigations of Electrical Breakdown in HV Polymeric Insulated Cables and Their Accessories Commonly used in Saudi Arabia", Final Report \# AR-15-46, King Abdulaziz City for Science and Technology (KACST), 2001. 
[4] L.A. Dissado and J.C. Fothergill, "Electrical Degradation and Breakdown in Polymers", Peter Peregrinus Ltd. UK, ISBN 0863411967, 1992.

[5] AEIC-CS5-1994, "Specification for Polyethylene and Crosslinked Polyethylene Insulated Shielded Power Cables rated 5-46 kV', 10th Edition, 1994.

[6] A.A. Al-Arainy, N.H. Malik, and M.I. Qureshi, "A Hybrid System of Long Term Accelerated Water Tree Testing of Cables at King Saud University", Proc. of $6^{\text {th }}$ Int'l Conference on Properties and Applications of Dielectric Materials", Xian, China, June 2000, pp. 550-553.

[7] J.A. Houlgreave; A. Eccles and J.C. Fothergill, "Characterization of Water Treeing and the Use of Frequency Acceleration Techniques", Proceedings of $6^{\text {th }}$ International Conference on Dielectric Materials, Measurements and Application, UK, pp. 492-495, 1992.

[8] A.A. Al-Arainy, A.A. Ahaidab, M.I. Qureshi, and N.H. Malik, "Statistical Evaluation of Water Tree Lengths in XLPE Cables at Different Temperatures", IEEE Trans. on Dielectrics and Electrical Insulation, Vol. 11, No. 6, pp. 9951004, 2004.

[9] T. Czaszejko "Statistical Analysis of Water Tree Lengths", Conference Record of the 1998 IEEE International Symposium on Electrical Insulation, Arlington, Virginia, USA., pp. 89-91, 1998.

[10] T. Czaszejko, "Water Tree Lengths and Their Distributions" Electrotechnika I Electronica. Vol. 18, No. 2, pp. 413-419, 1999. AGH Krakow (ISSN 0239-5274). 